\title{
Adaptive and unequal probability survey designs for environmental management
}

\author{
$\underline{\text { Jennifer A Brown }^{\mathrm{a}}}$, Mohammad Salehi M. ${ }^{\mathrm{b}}$, Mohammad Moradi ${ }^{\mathrm{c}}$, Bardia Panahbehagh ${ }^{\mathrm{d}}$ and David R Smith $^{\mathrm{e}}$ \\ ${ }^{a}$ Department of Mathematics and Statistics, University of Canterbury, Christchurch, New Zealand \\ Email: jennifer.brown@canterbury.ac.nz \\ ${ }^{b}$ Department of Mathematics, Statistics and Physics, Qatar University, Doha, Qatar \\ ${ }^{c}$ Department of Statistics, Razi University, Kermanshah, Iran \\ ${ }^{d}$ Department of Mathematics, Isfahan University of Technology, Isfahan, Iran \\ ${ }^{e}$ U.S. Geological Survey, Kearneysville, West Virginia, USA
}

\begin{abstract}
Designing an efficient large-area survey is a challenge, especially in environmental science when many populations are rare and clustered. Adaptive and unequal probability sampling designs are appealing when populations are rare and clustered because survey effort can be targeted to subareas of high interest. For example, higher density subareas are usually of more interest than lower density areas. Adaptive and unequal probability sampling offer flexibility for designing a long term survey because they can accommodate changes in survey objectives, changes in underlying environmental habitat, and changes in species-habitat models.
\end{abstract}

Keywords: Adaptive cluster sampling: Two-phase sampling: Stratified sampling: Adaptive two-stage sequential sampling: Complete allocation stratified sampling 
Brown et al., Adaptive and unequal probability sampling designs for environmental management

\section{INTRODUCTION}

The need for information for successful environmental management has led to an interest in developing new designs for statistical surveys. Environmental managers require information for monitoring current status and long term trends in environmental quality, and for assessing the impacts of changes in management practice. Examples include monitoring of protected areas such as national parks (Fancy et al. 2009), monitoring for biodiversity (Nielson et al. 2009), and designs to detect contamination (Olsen et al. 2009).

Some of the principle features of survey designs that provide good support for environmental management are designs that are robust for large areas, flexible and adaptable for heterogeneity, and responsive to change. Surveys must be capable of providing summary information on species occurrence, abundance, and structure collected from large areas of land or water. Large area surveys require a degree of spatial spread, or distribution of sample sites over the landscape, and this spread must be achieved in a statistical valid way to allow estimation of the population parameters of interest (Lister et al. 2009). Environmental systems are complex with a high degree of spatial and temporal heterogeneity in the underlying ecosystems. Surveys need to be flexible and adaptable to ensure they provide targeted information from such complex systems. Another important feature of environmental surveys is that because information needs and priorities change, surveys must be capable of responding to shifts in survey objectives.

Adaptive and unequal probability survey designs offer many attractive features for environmental surveys. Adaptive and unequal probability surveys can be designed for surveys of large areas providing the desired spatial spread and flexibility for responding to heterogeneity and changing priorities, while maintaining the key elements of probability-based statistical surveys. Adaptive sampling refers to sampling designs where the protocol for data-collection changes, evolves or adapts during the course of the survey. Unequal probability sampling is where the sample units (e.g., sample sites) have different probabilities of appearing in the sample (Thompson and Seber 1996).

There are many examples of adaptive sampling in environmental science and here we review some of them, with particular reference to populations that are rare and clustered. A text on adaptive sampling was published in 1996 by Thompson and Seber, which presents much of the theoretical background and development of estimators. The emphasis is on rare and clustered populations is simply because these are often the most challenging to survey and are also very common in environmental science (McDonald, 2004)

\section{ADAPTIVE CLUSTER SAMPLING}

Adaptive cluster sampling was introduced by Thompson (1990) and it was developed for sampling rare and clustered population. The design typically starts with a random sample, although it can also be applied to systematic sampling (Thompson 1991a; Acharya et al. 2000), stratified sampling (Thompson 1991b; Brown 1999) and two-stage sampling (Salehi and Seber 1997).

Prior to sampling, a threshold value is chosen, $C$, and if any of the units in the initial sample meet or exceed this threshold, $y_{i} \geq C$, then neighboring units are sampled. If any of these neighboring units meet this condition, their neighboring units are selected and so on. As sampling continues for any cluster that is detected in the initial sample, the shape and size of the cluster can be described.

The final sample is the collection of clusters that were detected in the initial sample, including any of the sample units that were in the initial sample but below the threshold. Survey effort is targeted to searching in the neighborhood of the location of where any plant (or animal) that is found in the initial sample. This feature of the design is very appealing. The design uses the intuitive behavior of a field biologist that once a rare plant is found they want to search in the immediate neighborhood, and puts this behavior in the framework of (unequal) probability sampling.

Horvitz-Thompson estimators are used for the population parameters in adaptive cluster sampling (Thompson and Seber 1996). Two terms need to be defined to help understand the terminology to distinguish "networks" and "clusters". A network is the collection of units around the unit in the initial sample that triggered neighborhood searching. All these units will have met the condition.

Neighborhood searching is an adaptive process, and for neighborhood searching to stop, units must have been measured and their value found to be below the threshold. These units are called "edge units". Together, networks and edge units make up a cluster. Any unit in the initial sample that does not meet the condition is considered a network of size one. 
Brown et al., Adaptive and unequal probability sampling designs for environmental management

In the example in figure 1 of blue-winged teal (Smith et al. 1995) an initial sample of 10 quadrants is taken (left side of figure). The survey was designed with the threshold condition, $y_{i} \geq 1$ and the definition of the neighborhood as the four surrounding quadrants. Only one quadrant in the initial sample triggered adaptive selection of the surrounding quadrants.

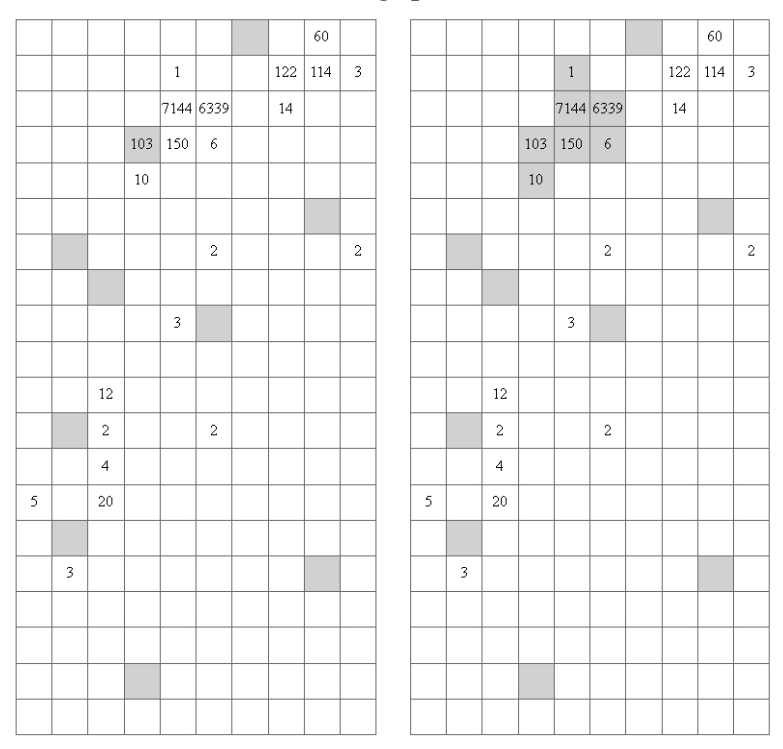

The final sample size is 16 (right side of figure), but in total, many more than 16 units had to be visited: the four neighboring units are always visited or checked if ducks are present, but only those in the initial sample or in a network are used in calculating the sample estimators. These other units are the edge units, and are visited and checked so that the "edge" of the networks can be defined. With a simple condition like $y_{i} \geq 1$, these units only needed to be checked to see if ducks are present or absent. However, when the condition is that the sample unit has a value that is larger than a count then the unit would need to be enumerated to check if the condition were met.

Software packages can be used to assist with these calculations, e.g. SAMPLE at http://www.lsc.usgs.gov/aeb/davids/acs/ (Morrison et al 2008).

Figure 1 Adaptive cluster sampling of blue-winged teal.

The efficiency of adaptive cluster sampling depends firstly on how clustered the population is and, secondly, on the survey design. As a general principle, the more clustered the population is, the more efficient adaptive cluster sampling is compared with simple random sampling. The design choices in adaptive cluster sampling are the sample unit size and shape, the size of the initial sample, the criteria for adaptive selection, and the neighborhood definition (e.g. the surrounding 2, 4 or 8 neighboring units). There is considerable literature on how to design an efficient survey and much of this is reviewed in Smith et al. (2004), and Turk and Borkowski (2005). A general principle is that efficient designs will be where the final sample size is not excessively larger than the initial sample size, and which has small networks. This can be achieved by using large criteria for adapting and small neighborhood definitions (Brown 2003).

An issue often raised with adaptive cluster sampling is that the size of the final sample is not known prior to sampling and this can make planning the field work difficult. Restricting the final sample size by a stopping rule has been discussed by Brown and Manly (1998), Salehi and Seber (2002), and Lo et al. (1997). Another approach is an inverse sampling design, where surveying stops once a set number of nonzero units have been selected (Christman and Lan 2001; Seber and Salehi 2004).

Adaptive cluster sampling has been used in a range of environmental situations. Some recent examples are the use of adaptive cluster sampling for surveys of plants (Philippi 2005), waterfowl (Smith et al. 1995), seaweed (Goldberg et al. 2006), shellfish (Smith et al. 2003), marsupials (Smith et al. 2004), forests (Talvitie et al. 2006; Magnussen et al. 2005), herpetofauna (Noon et al. 2006), larval sea lampreys (Sullivan et al. 2008), sediment load in rivers (Arabkhedri 2010), in hydroacoustic surveys (Conners and Schwager 2002) and fish eggs (Smith et al. 2004; Lo et al. 1997).

\section{STRATIFIED AND TWO-STAGE SAMPLING}

Adaptive sampling can also be used with conventional stratified and two-stage sampling. Two-stage sampling and stratified sampling are related in that in both designs the study area is sectioned into strata or primary units. In stratified sampling all strata are selected while in two-stage sampling a selection of primary units is chosen. Sampling (in the second phase) is done by taking a sample of secondary units from the chosen primary units, noting that in stratified sampling all primary units are chosen.

Both stratified and two-stage sampling can be very useful for sampling rare and clustered populations in the same way that adaptive cluster sampling is. Even if the location and size of the species-clusters are not 
known, as long as their location can be approximated by some auxiliary information and species-cluster size can be approximated by existing biological knowledge, survey effort can be targeted. One useful measure for approximating species-cluster location is a habitat suitability score estimated from environmental resource selection functions (Boyce et al. 2002) or from habitat modeling. This suitability score can be used to delineate primary units with differing levels of propensity for species occupancy. Survey effort can then be allocated among primary units targeting effort to the primary units or strata that are thought to be most likely to contain the clusters of interest. Even in situations where habitat maps and habitat prediction models for species distribution have some uncertainty, sectioning the study area into strata and primary units should be based on the idea of minimizing the within-primary unit or the within-stratum variance so that the units within the primary units are as similar as possible.

\subsection{Two-phase stratified sampling}

One of the early adaptive stratified designs was proposed by Francis (1984). In the two-phase stratified design the survey area is sectioned into strata and initial survey effort allocated based on the best available information using the standard approach of putting more effort in the more variable strata. After this initial phase of sampling, the preliminary information can be used to improve the estimate of the strata variability, and the remaining survey effort can be allocated to the strata that will be most effective in reducing the overall sample variance.

Using the first phase sample results to estimate within-stratum variance, the remaining sample units are added one by one to an individual stratum. At each step of this sequential allocation of sample units, the stratum that is allocated the unit is chosen on the basis of where the greatest reduction in variance will be. For some populations, rather than using within stratum variance of the criteria for adaptive allocation, the square of the stratum mean is preferred (Francis 1984). The final estimates are based on the pooled information from the first and second phases. This does result in a small bias, and bootstrapping has been proposed for bias correction and variance estimation (Manly 2004).

This adaptive allocation in the second phase is done to adjust or to make up for any shortcomings in the initial allocation of effort. As information is gained during the course of the survey, the design for datacollection evolves and adapts.

A similar scheme was proposed by Jolly and Hampton (1990). The design has been extended to surveying multiple populations (Manly et al. 2002). Smith and Lundy (2006) used a modified design to conduct a stratified sample of sea scallops. Based on the within-stratum mean from the first phase, a fixed amount of effort was allocated to each stratum where the mean was above a threshold value. They used the RaoBlackwell method (Thompson and Seber 1996) to derive an unbiased estimate for the population.

\subsection{Adaptive two-stage sequential sampling}

Another example of adaptive allocation with two-stage sampling is adaptive two-stage sequential sampling (Brown et al. 2008). An initial sample is taken from selected primary units. Then, in the second phase, additional units are allocated to the primary units proportional to the number of observed units in that primary unit that exceed a threshold value $g_{i} \cdot \lambda$, where $g_{i}$ is the number of sampled units in the $i^{\text {th }}$ primary unit that exceed the threshold value and $\lambda$ is a multiplier.

\subsection{Complete allocation stratified sampling}

Another example of adaptive sampling applied to conventional stratified or two-stage sampling is a complete allocation stratified design (Salehi and Brown 2010). This is a simplified design for adaptive stratified sampling. If any unit in a stratum has a value that exceeds a threshold, the stratum is completely surveyed. It is simplified in two ways: the rule to decide on whether a stratum is to be allocated additional survey effort does not require the first-phase survey in the stratum to be completed. Secondly, the instruction to the field crew on how much additional effort is required is simply to survey the entire stratum.

The complete allocation stratified design merges the best features of some of the previous adaptive designs. In adaptive cluster sampling, the appeal is that it allows the field biologist to target survey effort to neighborhoods where individuals from a rare species were observed in the initial (first-phase) sample. This adaptive searching of the neighborhood in adaptive cluster sampling is similar to conducting a complete search in the vicinity of the found individual. In complete allocation, once an individual is observed, the 
Brown et al., Adaptive and unequal probability sampling designs for environmental management

neighborhood is completely searched. The difference is that in adaptive cluster sampling, the neighborhood is not defined prior to sampling and, for some populations, can be excessively large (Brown 2003). In complete stratified allocation, the searched neighborhood is defined and restrained by the stratum boundary.

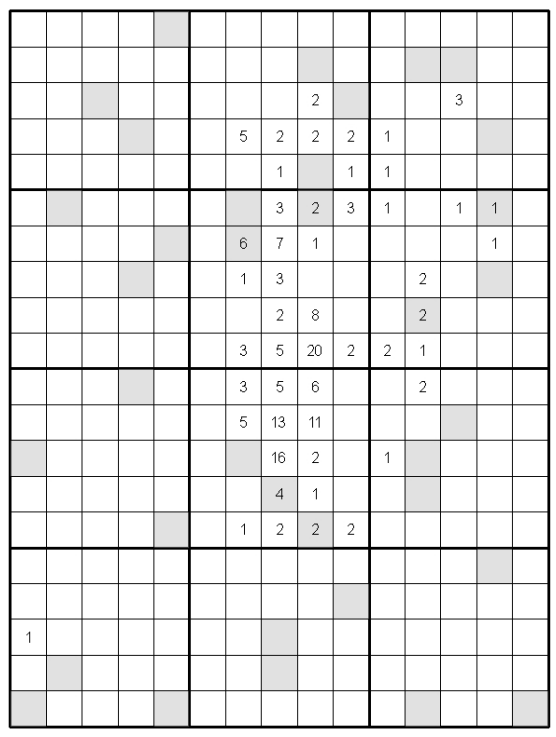

In the example in figure 2 of Castle Hill buttercups (Brown 2010) the study site was divided into 12 strata. In the first phase, a simple random sample of size 3 was taken from each of the stratum. Three strata had first-phase samples containing buttercups. In the second phase, these three strata were surveyed completely. The total final sample size is therefore $(3 \times 25)+9 \times 3)=102$.

In the simulation study of Salehi and Brown (2010) gains in efficiency over non-adaptive stratified sampling were for some designs, very large.

Figure 2 Complete allocation stratified sampling of buttercups

\section{DISCUSSION}

Adaptive and unequal probability sampling designs offer a wide range of flexible and useful survey techniques. These designs are especially useful for environmental sampling and for surveys of rare and clustered populations because they allow survey effort to be targeted to where any plant or animal of interest has been found. Changes to survey objectives can be readily accommodated by altering primary unit, or strata, boundaries, shifting first-phase allocation to effort among primary units, and changing the threshold condition used for second-phase adaptive allocation. Similarly, as the underlying environmental habitat changes, or as habitat models improve, the sample design can be modified. The flexibility in adaptive and unequal probability sampling is that population estimates derived from surveys where design features have changed (e.g., relative survey effort allocation and threshold condition) are still comparable. In long term environmental monitoring these two features of design flexibility and estimates that are comparable amongyears are important considerations.

Adaptive and unequal probability sampling can be applied to any sampling situation. We have discussed adaptive selection as a complement to simple random sampling, two-stage and stratified sampling. We use the terminology suggested by Salehi and Brown (2010) with the use of the terms "adaptive searching" and "adaptive allocation" to distinguish two categories. Adaptive searching refers to designs such as adaptive cluster sampling where the neighborhood is searched. In contrast, in adaptive allocation, extra effort is initiated once a collection of units are sampled (e.g. the stratum or the primary unit). The distinction between the two classes is based on where and when the decision to allocate extra effort can be made: immediately after an individual sample unit is measured or once a collection of units has been completely sampled.

In adaptive cluster sampling, extra effort is allocated for adaptive searching in the neighborhood of units in the initial sample that met the threshold condition. With stratified or two-stage designs such as two-phase stratified sampling (Francis 1984), adaptive two-stage sequential sampling (Brown et al 2008) and complete allocation stratified sampling (Salehi and Brown 2010), adaptive allocation of additional effort is triggered on the basis of a measure of the whole stratum (or primary unit).

All the designs discussed are remarkably efficient, giving estimates of populations that have lower variance than the conventional design without the adaptive selection. However, as with conventional sampling, the survey must be carefully designed to achieve these efficiencies. Design features include choice of the size and number of stratum, allocation of effort to the first phase (i.e. the initial sample before the additional effort 
Brown et al., Adaptive and unequal probability sampling designs for environmental management

is allocated) relative to second-phase effort, the threshold used to trigger adaptive allocation, and, for adaptive cluster sampling, the neighborhood definition.

It is important to ensure adequate effort is available for the adaptive allocation that occurs in the second phase of sampling if large gains in efficiency are to be realized. For the same amount of effort, Brown et al. (2008) recommend putting less effort into the initial sample of the selected primary sample units to ensure more effort is available for the sequential allocation of additional units, compared with the reverse. Another recommendation is that the threshold value that is used to "trigger" adaptive allocation of additional units should be relatively high. These recommendations are consistent with what is recommended for adaptive cluster sampling (Brown 2003, Smith et al. 2004).

For adaptive stratified and two-stage designs sample efficiency improved when the primary units created boundaries that encompassed aggregates or clusters. This is because the adaptive allocation of effort to primary units is a very effective way of targeting effort to where the species of interest is located. Similarly when stratification intensity increases with more, and smaller, primary units or strata, the survey efficiency improves as the primary units become closer to matching the size of the aggregates in the underlying population.

\section{ACKNOWLEDGEMENTS}

Thank you to Miriam Hodge for ongoing collaborative work in this field.

\section{REFERENCES}

Acharya, B. Bhattarai, G. de Gier, A. and Stein, A. (2000). Systematic adaptive cluster sampling for the assessment of rare tree species in Nepal. Forest Ecology and Management, 137: 65-73.

Arabkhedri, M. Lai, F.S. Noor-Akma, I. and Mohamad-Roslan, M.K. (2010). An application of adaptive cluster sampling for estimating total suspended sediment load. Hydrology Research, 41(1): 63-73.

Boyce, M.S. Vernier, P.R. Nielson, S.E. and Schmiegelow, F.A.K. (2002). Evaluating resource selection functions. Ecological Modelling, 157: 281-300.

Brown, J.A. (2010). Adaptive sampling of ecological populations. In: Yue Rong (ed) Environmental Statistics and Data Analysis. ILM Publications, Hertfordshire, UK, (in press).

Brown, J.A. (1999). A comparison of two stratified sampling designs: adaptive cluster sampling and a twophase sampling design. Australia and New Zealand Journal of Statistics, 41(4): 395-404.

Brown, J.A. (2003). Designing an efficient adaptive cluster sample. Environmental and Ecological Statistics, 10: $95-105$.

Brown, J.A. and Manly, B.F.J. (1998). Restricted adaptive cluster sampling. Environmental and Ecological Statistics, 5: 47-62.

Brown, J.A. M. Salehi, M. Moradi, M. Bell, G. and Smith, D.R. (2008). An adaptive two-stage sequential design for sampling rare and clustered populations. Population Ecology, 50(3): 239-245.

Christman, M.C. and Lan, F. (2001). Inverse adaptive cluster sampling. Biometrics, 57: 1096-1105.

Cochran, W.G. (1977). Sampling Techniques. 3rd edition. Wiley, New York.

Conners, M.E. and Schwager, S.J. (2002). The use of adaptive cluster sampling for hydroacoustic surveys. Journal of Marine Science: Journal du Conseil, 59(6): 1314-1325.

Fancy, S.G. Gross, J.E. and Carter, S.L. (2009). Monitoring the condition of natural resources in US national parks. Environmental Monitoring and Assessment, 151: 161-174.

Francis, R.I.C.C. (1984). An adaptive strategy for stratified random trawl surveys. New Zealand Journal of Marine and Freshwater Research, 18: 59-71.

Goldberg, N.A. Heine, J.N. and Brown, J.A. (2006). The application of adaptive cluster sampling for rare subtidal macroalgae. Marine Biology, 151: 1343-1348.

Horvitz, D.G. and Thompson, D.J. (1952). A generalization of sampling without replacement from a finite universe. Journal of the American Statistical Association, 47: 663-685.

Jolly, G.M. and Hampton, I. (1990). A stratified random transect design for acoustic surveys of fish stocks. Canadian Journal of Fisheries and Aquatic Sciences, 4: 1282-1291.

Lister, A.J. Scott, C.T. and Scott, C.T. (2009). Use of space-filling curves to select sample locations in natural resource monitoring studies. Environmental Monitoring and Assessment, 149:71-80.

Lo, N.C.H. Griffith, D. and Hunter, J.R. (1997). Using restricted adaptive cluster sampling to estimate Pacific hake larval abundance. California Cooperative Oceanic Fisheries Investigations Report 37:160-174. 
Brown et al., Adaptive and unequal probability sampling designs for environmental management

McDonald, L.L. (2004). Sampling rare populations. In: Thompson, W.L. (ed) Sampling Rare and Elusive Species. Island Press, Washington DC, p. 11-42.

Magnussen, S. Kurz, W. Leckie, D.G. and Paradine, D. (2005). Adaptive cluster sampling for estimation of deforestation rates. European Journal of Forest Research, 124(3): 207-220.

Manly, B.F.J. (2004). Using the bootstrap with two-phase adaptive stratified samples from multiple populations at multiple locations. Environmental and Ecological Statistics, 11:367-383.

Manly, B.F.J. Akroyd, J.M. and Walshe, K.A.R. (2002). Two-phase stratified random surveys on multiple populations at multiple locations. New Zealand Journal of Marine and Freshwater Research, 36: 581-591.

Morrison, L.W. Smith, D.R. Nichols, D.W. and Young, C.C. (2008). Using computer simulations to evaluate sample design: an example with the Missouri bladderpod. Population Ecology, 50: 417-425.

Nielsen, S.E. Haughland, D.L. Bayne, E. and Schieck, J. (2009). Capacity of large-scale, long-term biodiversity monitoring programmes to detect trends in species prevalence. Biodiversity Conservation, 18:2961-2978.

Noon, B.R. Ishwar, N.M. and Vasudevan, K. (2006). Efficiency of adaptive cluster and random sampling in detecting terrestrial herpetofauna in a tropical rainforest. Wildlife Society Bulletin, 34: 59-68.

Olsen, A.R. Snyder, B.D. Stahl, L.L. and Pitt, J.L. (2009). Survey design for lakes and reservoirs in the United States to assess contaminants in fish tissue. Environmental Monitoring and Assessment, 150:91-100.

Philippi, T. (2005). Adaptive cluster sampling for estimation of abundances within local populations of lowabundance plants. Ecology, 86: 1091-1100.

Salehi, M.M. and Seber, G.A.F. (1997). Two-stage adaptive cluster sampling. Biometrics, 53: 959-970.

Salehi, M.M. and Seber, G.A.F. (2002). Unbiased estimators for restricted adaptive cluster sampling. Australian and New Zealand Journal of Statistics, 44: 63-74.

Salehi, M. and Brown, J.A. (2010). Complete allocation sampling: An efficient and easily implemented adaptive sampling design. Population Ecology, (in press).

Seber, G.A.F. and Salehi, M.M. (2004). Adaptive sampling. In: Armitage, P. Colton, T. (eds) Encyclopedia of Biostatistics Volume 1, 2nd ed. Wiley and Sons, Chichester, p.59-65.

Smith, D.R. Brown, J.A. and Lo, N.C.H. (2004). Application of adaptive cluster sampling to biological populations. In: Thompson WL (ed) Sampling Rare and Elusive Species. Island Press, Washington DC, p. 75-122.

Smith, D.R. Conroy, M.J. and Brakhage, D.H. (1995). Efficiency of adaptive cluster sampling for estimating density of wintering waterfowl. Biometrics, 51: 777-788.

Smith, D.R. Villella, R.F. and Lemarié, D.P. (2003). Application of adaptive cluster sampling to low-density populations of freshwater mussels. Environmental and Ecological Statistics, 10: 7-15.

Smith, S.J. and Lundy, M.J. (2006). Improving the precision of design-based scallop drag surveys using adaptive allocation methods. Canadian Journal of Fisheries and Aquatic Sciences, 63: 1639-1646.

Sullivan, W.P. Morrison, B.J. Beamish, F.W.H. (2008). Adaptive cluster sampling: estimating density of spatially autocorrelated larvae of the sea lamprey with improved precision. Journal of Great Lakes Research, 34: 86-97.

Talvitie, M. Leino, O. and Holopainen, M. (2006). Inventory of sparse forest populations using adaptive cluster sampling. Silva Fennica, 40: 101-108.

Thompson, S.K. (1990). Adaptive cluster sampling. Journal of the American Statistical Association, 85: 1050-1059.

Thompson, S.K. (1991a). Adaptive cluster sampling: Designs with primary and secondary units. Biometrics, 47: 1103-1115.

Thompson, S.K. (1991b). Stratified adaptive cluster sampling. Biometrika, 78: 389-397.

Thompson, S.K. and Seber, G.A.F. (1996). Adaptive Sampling. Wiley, New York.

Turk, P. and Borkowski, J.J. (2005). A review of adaptive cluster sampling: 1990-2003. Environmental and Ecological Statistics, 12: 55-94. 